\title{
In-flight calibration of the HIFI diplexers
}

\author{
Michael Mueller • Willem Jellema • \\ Bertrand Delforge $\cdot$ David Teyssier · \\ Martin Eggens • Robert Huisman • \\ Geert Keizer
}

Received: 26 September 2013 / Accepted: 9 December 2013 / Published online: 16 January 2014

(C) The Author(s) 2014. This article is published with open access at Springerlink.com

\begin{abstract}
HIFI is a heterodyne spectrometer aboard the Herschel Space Observatory, providing high-spectral-resolution capabilities. Of its seven frequency bands, four (bands 3, 4, 6, and 7) employ Martin-Puplett diplexers to combine sky signal and local oscillator at the two linear polarizations $\mathrm{H}$ and $\mathrm{V}$, prior to feeding them into the mixers (receivers). The optical path difference in each of these 8 diplexers must be tuned to the observed frequency. The required actuator currents were determined in flight before the start of routine science observations. We here report on regular (roughly quarterly) engineering test observations to validate the repeatability of the HIFI diplexers during the routine phase of Herschel operations. We find the optical path difference to be stable to within $0.4 \%$ of the relevant wavelength, typically at the sub-micron level. We conclude that the repeatability and precision of the diplexer tuning mechanism are so high that science data are in no way negatively affected. With the diplexer calibration established and validated, this line of reasoning can be reversed, and the diplexers can be used as relative spectrometers to measure the localoscillator frequency, i.e., to check the spectral purity of the local oscillator across the diplexer bands. This was done from before launch out to the last months of cryogenic operations in space.
\end{abstract}

Keywords Herschel space observatory $\cdot$ HIFI · Heterodyne $\cdot$ Far-infrared · Instrumentation · Calibration

M. Mueller $(\varangle) \cdot$ W. Jellema · B. Delforge · M. Eggens · R. Huisman · G. Keizer SRON Netherlands Institute for Space Research,

Postbus 800, 9700 AV Groningen, The Netherlands

e-mail:m.mueller@sron.nl

D. Teyssier

European Space Astronomy Centre, ESA,

PO Box 78, 28691 Villanueva de la Cañada, Madrid, Spain 


\section{Introduction}

HIFI (Heterodyne Instrument for the Far-Infrared, [2]) is the high-spectral-resolution instrument aboard the Herschel Space Observatory [6]. It provides nearly continuous frequency coverage over the range from 480 to $1910 \mathrm{GHz}$ in seven bands (bands 1-7). HIFI's high spectral resolution is obtained through heterodyning: the high-frequency sky signal is combined with a tunable monochromatic reference frequency (local oscillator, LO) in non-linear mixers. After filtering, this results in an intermediatefrequency (IF) signal at the beat frequency $f_{\mathrm{IF}}$ between sky signal and LO, which is fed into spectrometers covering IF ranges from $f_{\mathrm{IF}} \sim 4-8 \mathrm{GHz}$ in bands $1-5$ and $f_{\mathrm{IF}} \sim 2.4-4.8 \mathrm{GHz}$ in bands 6 and 7 . For each LO frequency $f_{\mathrm{LO}}, \mathrm{HIFI}$ is sensitive to sky frequency bands of $f_{\mathrm{LO}}+f_{\mathrm{IF}}$ (upper sideband) and $f_{\mathrm{LO}}-f_{\mathrm{IF}}$ (lower sideband). There are two independent mixers per band, each sensitive to one linear polarization (denoted in the following as $\mathrm{H}$ and $\mathrm{V}$ for horizontal and vertical), and each feeding into an independent IF signal chain.

Before entering the mixers, sky signal and LO radiation must be combined with one another, then split by linear polarization. Two different solutions are used within HIFI: beam splitters in bands 1, 2, and 5; diplexers in bands 3, 4, 6, and 7. Beam splitters have no moving parts and a very constant bandpass over the IF range. In return, more than $90 \%$ of the LO power is discarded. In the diplexer bands, the available LO power did not allow the use of beam splitters. These bands employ Martin-Puplett interferometers [4] (referred to as "diplexers" in the following; see Section 2), which use practically all LO power but require in-flight tuning to the appropriate optical path difference (OPD) to obtain optimum coupling for the frequencies of interest.

HIFI's eight diplexers share a common layout, in which an actuator moves a rooftop mirror to tune the OPD. Tuning is done in open loop by simply commanding an actuator current. For routine science observations, the required actuator current is taken from a look-up table that is part of the Common Uplink System (CUS). The final diplexer calibration had to be done under zero-gravity conditions, i.e., in flight [7]. The calibration was established during the commissioning phase, repeating the diplexer scans done during the pre-launch thermal-balance thermalvacuum (TBTV) tests described by [1]. To validate the repeatability of the diplexer calibration, diplexer scans were repeated regularly, roughly quarterly.

The main topic of this manuscript is to describe the HIFI diplexer test observations done in flight and their results. We start with a quick overview of the diplexer layout as needed for our purposes, followed by a description of the diplexer scan observations, and by a summary of their results. In the final section, we describe the use of HIFI's diplexers as tools to detect spectral impurities of the LO.

\section{Layout of the HIFI diplexers}

The optical path of a diplexer band is depicted in Fig. 1. A first polarizing grid combines LO and sky signal, which enter at right angles. The two beams leaving the entrance grid contain a mixture of LO and sky signal at orthogonal linear polarization from one another (one has passed the polarizer in transmission, the other in 


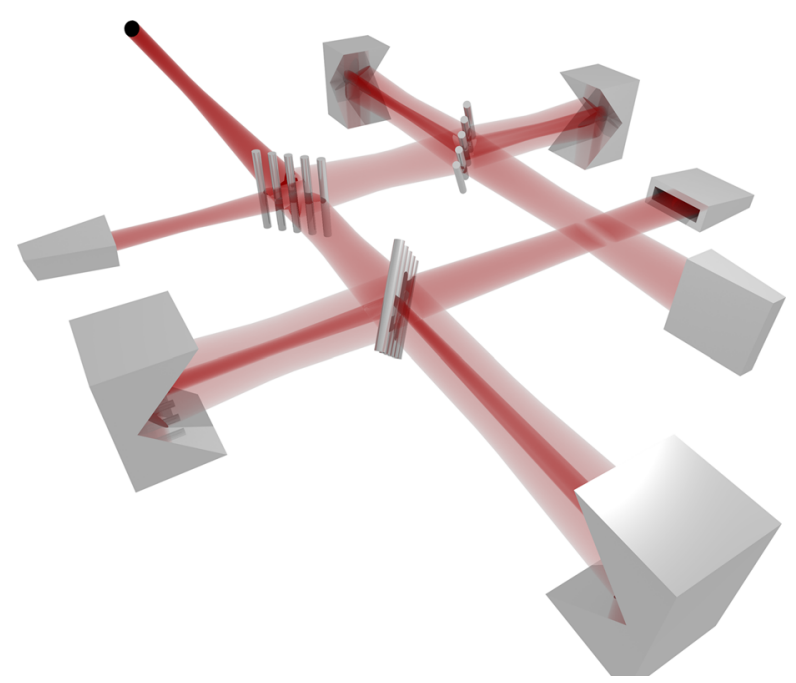

Fig. 1 Sketch of the optical path of a HIFI diplexer band. Radiation enters from the upper left corner (sky signal: black circle; LO: stylized horn antenna) and is directed to the two mixers at polarizations $\mathrm{H}$ and $\mathrm{V}$ (depicted as horn antennas on the right-hand side of the Figure). A polarizing grid combines sky and LO and sends two beams to the two diplexers (upper and lower corner of the Figure), each consisting of a polarizing grid and two rooftop mirrors. Before entering the diplexers, the beams are a combination of linearly polarized sky $(\mathrm{H}$ and $\mathrm{V})$ and linearly polarized $\mathrm{LO}(\mathrm{V}$ and $\mathrm{H})$. The goal is to align their polarizations by properly tuning the diplexers. The diplexer layout is depicted in Fig. 2

reflection). Each beam is sent to a diplexer, in which the polarization of LO and sky signal is aligned, at minimum coupling loss, before being fed into the (polarized) mixers.

The eight diplexers (four bands times two polarizations) all follow the same general Martin-Puplett layout [4], a variant of the Michelson-Morley interferometer. They consist of a polarizing grid oriented at $45^{\circ}$ and two rooftop mirrors (see Fig. 2). The polarizer produces two linearly polarized beams containing LO and sky signal. Each is reflected off a rooftop mirror that is oriented such that it rotates the polarization by $90^{\circ}$. At a second pass through the polarizer, the two beams are therefore coupled to the output where they interfere with one another. ${ }^{1}$ The polarization of the output beam at frequency $f$ depends on the phase shift $\phi$ induced by the optical path difference (OPD):

$$
\phi=2 \pi \mathrm{OPD} \frac{f}{c} .
$$

In general, the output beam has elliptical polarization. The output power $P(f)$ at the polarization orthogonal to that of the input depends on the phase shift as follows:

$$
P(f) \propto 1+\cos \phi \propto \cos ^{2} \frac{\phi}{2} .
$$

\footnotetext{
${ }^{1}$ In practice, the polarizers and other optical components suffer from imperfections, leading to standing waves and polarization crosstalk, among other things. This can be neglected for our purposes, though.
} 


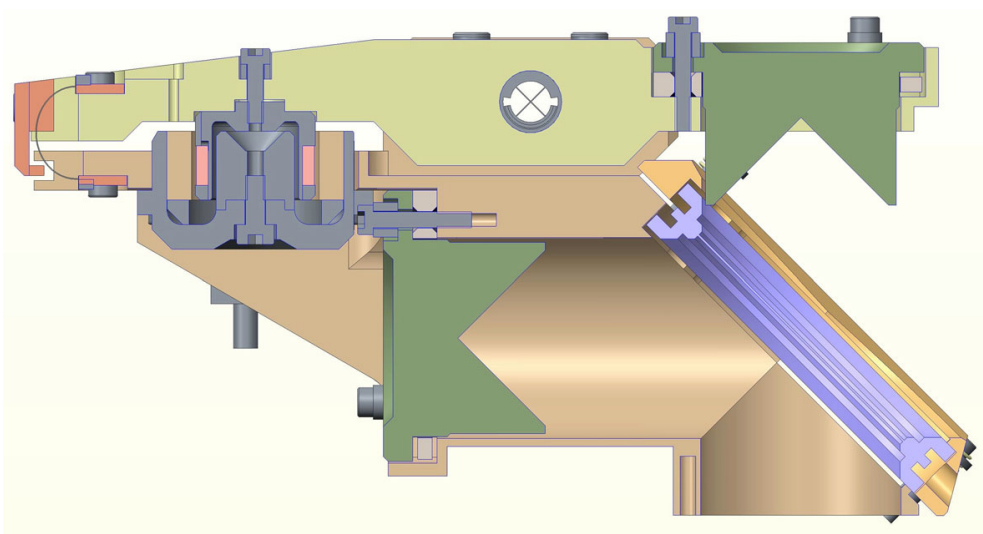

Fig. 2 Cross-section of a HIFI diplexer: the polarizer (at $45^{\circ}$ ) and the two rooftop mirrors are on the right-hand side. The upper rooftop mirror is attached to a lever that extends to the left edge of the Figure. The lever is connected to the rest of the structure through two flexural pivots (one is depicted as a black cross above the center of the Figure) that allow it to rotate about an axis perpendicular to the plane of this Figure. The pivoting angle is limited by two endstops visible at the left edge. A voice-coil actuator on the left-hand side of the lever can apply a force that acts against the spring constant of the pivots, causing the lever to pivot and the rooftop mirror to move

Special cases occur for $\phi=2 n \pi$ (output beam linearly polarized, orthogonal to input beam) and for $\phi=(2 n+1) \pi$ (output beam linearly polarized, aligned with input beam), for integer values of $n$. The LO and sky signal enter the diplexer at mutually orthogonal polarization, the target phase shifts are therefore

- $f_{\mathrm{LO}}: \phi=2 n \pi$

$-f_{\mathrm{LO}} \pm f_{\mathrm{IF}}: \phi=(2 n \pm 1) \pi$.

Optimally, these conditions should be met for the center of the IF range of $6 \mathrm{GHz}$ (bands 1-5) or $3.6 \mathrm{GHz}$ (bands 6 and 7). However, this problem is overdetermined (three conditions to be met by two variables: OPD and $n$ ). The sky-signal condition implies that $\phi$ should equal $2 \pi$ between the upper and the lower sideband. With the central IF stated above and Eq. 1, this means that the nominal OPD should be $\frac{c}{2 f_{\mathrm{IF}}}$, equaling

- $\quad 25 \mathrm{~mm}$ in bands 3 and 4

- $\quad \sim 41.7 \mathrm{~mm}$ in bands 6 and 7 .

These values being large compared to the wavelength (hundreds of $\mu \mathrm{m}$ ), the diplexer is tuned to an OPD as close as possible to the nominal value, such that the LO coupling is optimum. In general, this means that the sky signal is coupled optimally at a frequency that is somewhat offset from the center of the IF range.

The OPD is set by moving one of the two rooftop mirrors. As seen in Fig. 2, the movable rooftop mirror is attached to a lever (lever arm length $L=27.5 \mathrm{~mm}$ ). Rotation of the lever is possible thanks to the use of flexural pivots; the mechanical design is such that the lever cannot rotate about any other axis. A voice-coil actuator 
on the far side of the lever applies a force that acts against the spring constant of the pivots. By design, the slight rotation of the rooftop mirrors leaves the OPD invariant.

The resulting minimum step size of the rooftop mirror is $<200 \mathrm{~nm}$ over a range of approximately $550 \mu \mathrm{m}$. $L$ is long compared to the linear throw of the actuator. The mirror displacement equals OPD/2 (radiation travels back and forth between polarizer and rooftop mirror) and is well approximated by a linear function of actuator current $I$, with a small quadratic correction term:

$$
\frac{\mathrm{OPD}}{2}=d_{0}+\frac{\pi L}{180^{\circ}}\left(\alpha I^{2}+\beta I\right)
$$

with zero-current offset $d_{0}$ and the constants $\alpha$ and $\beta$ relating actuator current to the pivoting angle. $\beta$ is a measure of the actuator response divided by the spring constant of the pivot. In order to obtain the nominal OPD (see above) close to zero current, design values for $d_{0}$ are $12.5 \mathrm{~mm}$ and $20.83 \mathrm{~mm}$ in bands $3+4$ and bands $6+7$, respectively.

\section{Diplexer scan observations}

The diplexer calibration was based on test observations done during in-orbit commissioning (CoP); they were repeated regularly, roughly quarterly, to verify reproducibility. In each of the four diplexer bands, the LO was tuned to a number of pre-defined frequencies sampling the band's frequency range. At each LO frequency, the mixer was biased such that it can remain stable at all diplexer positions. ${ }^{2}$ The actual diplexer scan consists in scanning the diplexer actuator current through its allowable range and measuring the resulting mixer current as a measure of coupling. For efficiency, the scans are done quickly relative to the time constant of the actuator current source. This results in an offset between commanded and actual actuator current at the time of mixer readout. The current range was therefore covered twice, in "forward" and "reverse" directions, so this offset can be corrected for.

The OPD is roughly a linear function of actuator current (see Eq. 3), and the mixer current is roughly a linear function of input flux (with a non-zero axis offset). Plots of the mixer current as a function of actuator current should therefore display a sinusoidal interference pattern (see Eq. 2). Indeed, this is observed (see Fig. 3 for two sample scans).

\subsection{Full surveys up to cycle 55}

The AOT HifiEng_Diplexer_calibration_vs_D2_COP was used between commissioning (CoP) and cycle 55 (Dec 2011). These observations replicate the pre-launch diplexer scans described in [1]. The diplexer scans are done at some 20 LO frequencies per band. For each LO frequency, the scan is done for a series of

\footnotetext{
${ }^{2}$ This is doable with nominal biases in bands $3+4$. However, for bands $6+7$, this means biasing in the resistive regime to avoid oscillation when the mixer is essentially unpumped as the diplexer gets detuned.
} 
Diplexer scan 1342266910; mixer band 3 polar $\mathrm{H}$ LO band 3b@953.00 GHz; VD2set (avg)=2.845 V; forward

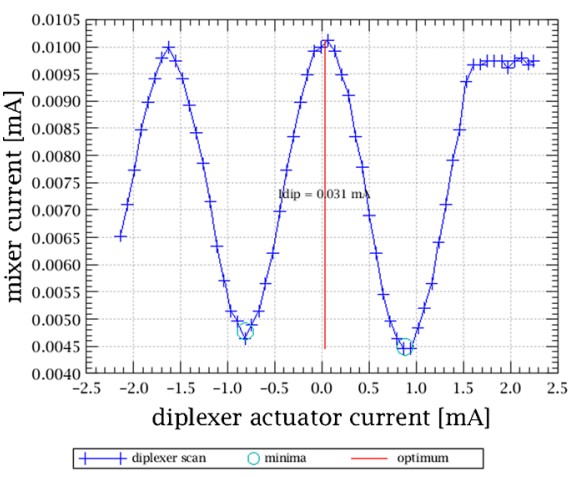

Diplexer scan 1342263041; mixer band 7 polar V LO band $7 \mathrm{~b} @ 1893.00 \mathrm{GHz}$; VD2set (avg)=2.401 v ; reverse

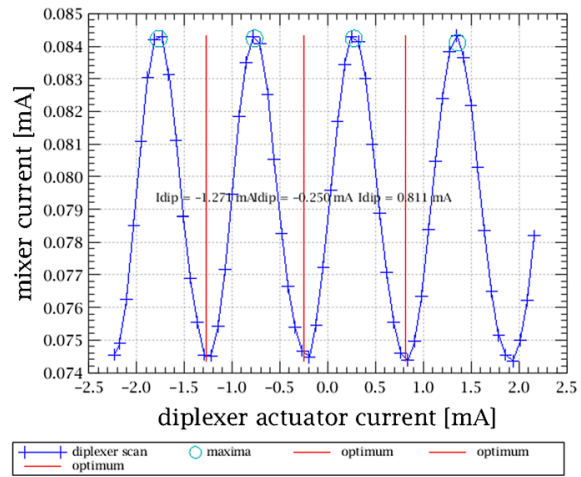

Fig. 3 Two sample diplexer scans: mixer current (coupling) as a function of actuator current (OPD) for two HIFI frequencies: $953 \mathrm{GHZ}$ (left) and $1893 \mathrm{GHz}$ (right). Note the shorter wavelength in band 7. In band 3 (left), the actuator runs into an endstop at the largest actuator currents, hence the flat asymptote

LO power settings (which is controlled by the drain voltage $\mathrm{Vd} 2$ of the 2 nd power amplifier in the chain): 5 settings in bands 3 and 4, 7 settings in bands 6 and 7 .

Seven complete sets of such diplexer surveys were done in orbit (see Table 1), each lasting $\sim 6 \mathrm{~h}$.

Table 1 Observation IDs of the diplexer surveys considered in this work

\begin{tabular}{lllll}
\hline & Band 3 & Band 4 & Band 6 & Band 7 \\
\hline QSFT-0 (HIFI CoP) & 1342189622 & 1342189639 & 1342189646 & 1342189631 \\
QSFT-1 (cycle 13) & 1342196499 & 1342196446 & 1342196528 & 1342196556 \\
QSFT-2 (cycles 23, 24) & 1342206446 & 1342206380 & 1342205463 & 1342205464 \\
QSFT-3 (cycles 28, 31, 32) & 1342210658 & 1342213328 & 1342213152 & 1342210783 \\
QSFT-4 (cycles 39, 40) & 1342221401 & 1342221402 & 1342221404 & 1342220454 \\
QSFT-5 (cycles 47, 48) & 1342227388 & 1342227389 & 1342227525 & 1342229757 \\
QSFT-6 (cycle 55) & 1342235006 & 1342235008 & 1342235057 & 1342235083 \\
QSFT-7 (cycle 66) & 1342246040 & 1342246334 & 1342246054 & 1342245994 \\
QSFT-8 (cycle 72) & 1342249555 & 1342249620 & 1342249406 & 1342249557 \\
QSFT-9 (cycle 78) & 1342254367 & 1342254356 & 1342254397 & 1342254890 \\
QSFT-10 (cycle 84) & 1342262662 & 1342263039 & 1342263040 & 1342263041 \\
QSFT-11 (cycle 90) & 1342270443 & 1342270444 & 1342270445 & 1342270446 \\
\hline
\end{tabular}

Full diplexer surveys are given in the first seven rows (QSFT 0-6; see Section 3.1); later surveys are of the shortened kind (see Section 3.2). For reference, we provide the cycle number in which the tests were executed (Herschel scheduling was organized in cycles of 14 days. Routine science observations ended towards the end of cycle 90 with the depletion of the on-board cryogen). QSFT-0 took place during HIFI commissioning $(\mathrm{CoP})$. 


\subsection{Shortened diplexer surveys in cycles $66+$}

The above-mentioned full surveys showed an excellent diplexer repeatability (see below). Because of this, the HIFI ICC devised a much shorter survey scheme for the last 5 rounds, AOT HifiEng_Diplexer_calibration_short_COP. As in regular science observations (and unlike the previous diplexer surveys), the LO is tuned autonomously $(\mathrm{Vd} 2$ is optimized autonomously for maximum mixer current), so the scan along the $\mathrm{Vd} 2$ axis is no longer needed. Also, the number of $\mathrm{LO}$ frequencies is reduced to 2 per $\mathrm{LO}$ subband (there are two LO subbands per mixer).

These shortened tests took only some $0.6 \mathrm{~h}$ to complete, i.e., an order of magnitude less than the full surveys. Between the 5 shortened diplexer surveys, more than a day's worth of spacecraft time was saved.

\section{Data analysis}

HIPE scripts that are part of the ICC distribution were used to retrieve the relevant housekeeping data (mixer current, actuator current, LO frequency, ... ) for all diplexer scan observations given in Table 1. Dedicated MATLAB routines were then used to fit the observed mixer current as a function of actuator current.

In a first step, the coupling maxima (that correspond to optimum coupling) are determined for each LO frequency (see Fig. 3). In practice, however, the shape of the coupling curve is somewhat distorted around the maxima, leading to position offsets. This is due to problems that scale with coupled LO power, such as standing waves, transient $\mathrm{LO}$ instabilities, ${ }^{3}$ crosstalk between the $\mathrm{H}$ and $\mathrm{V}$ diplexers, or spectral impurities. We therefore fit the position of the minima, and take the maxima to be halfway between adjacent minima. This approach was validated before launch, see [1].

From the inferred optimum-coupling currents as a function of LO frequency, we then obtain diplexer calibration constants for each test observation, mixer, and polarization. Specifically, two parameters are fitted: the zero-current offset $d_{0}$ and the pivoting angle per actuator current $\beta$ (see Eqs. 2 and 3). The quadratic coefficient, $\alpha$, is taken to be proportional to $\beta$. This is justified by pre-launch tests, in which $\alpha$ and $\beta$ were measured for all diplexers, both at room temperature and at cryogenic temperature (4 K). It was found that the ratio $\alpha / \beta$ did not change with temperature, for all diplexers.

In the fitting, data points with non-optimal mixer pumping or $\mathrm{LO}$ frequencies with known spectral impurities (see Section 5) were discarded so as to not skew the results. Results on a per-obsID basis are given in Table 2 .

Look-up table used in-flight (CUS) The in-flight diplexer calibration was established from the diplexer scans obtained during CoP. It was used to generate look-up tables

\footnotetext{
${ }^{3}$ The LO requires some stabilization time after each tuning. This is accounted for in the design of science observations but not in the diplexer scans (which would have taken prohibitively long otherwise).
} 


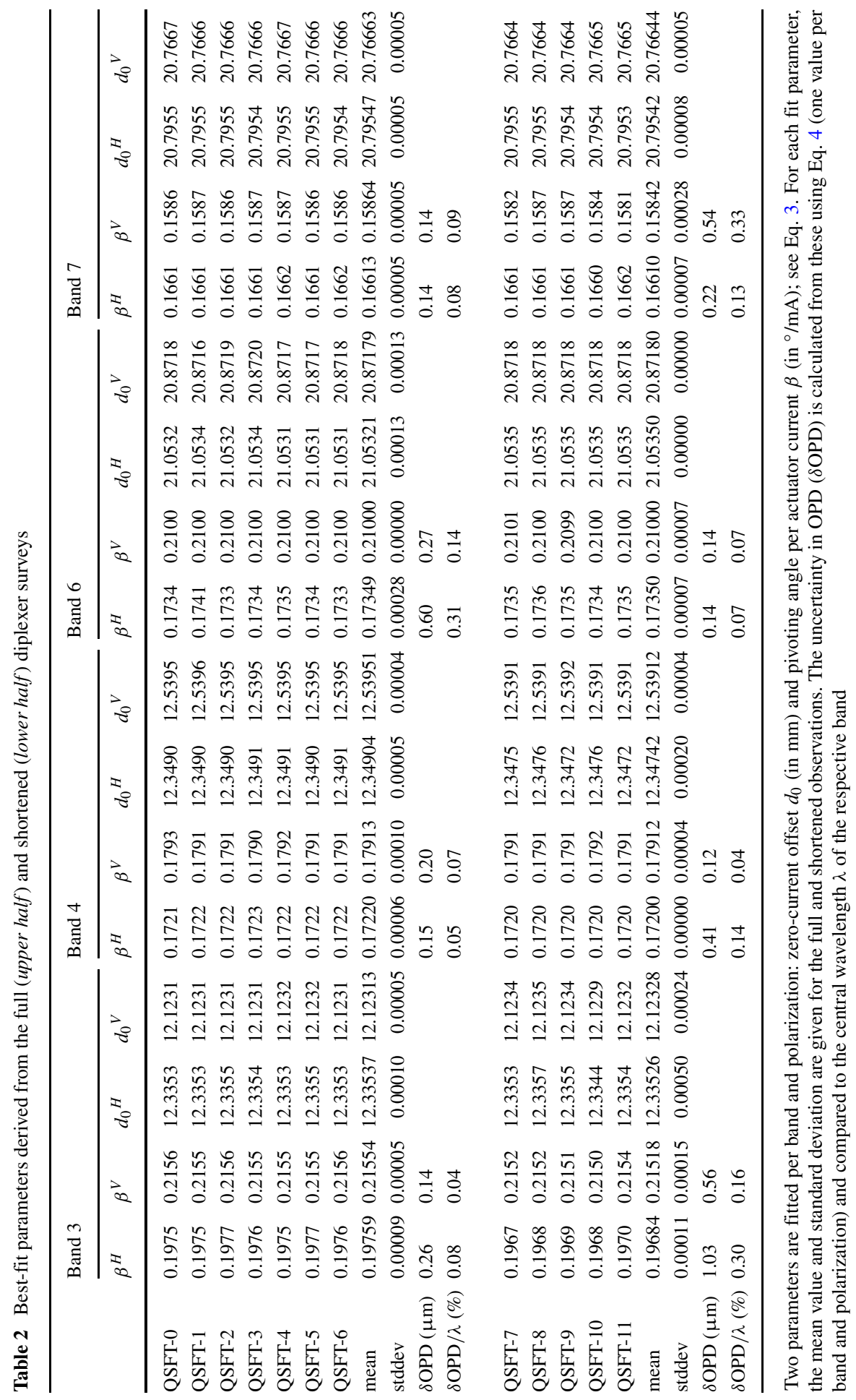


(one per diplexer) of required actuator current as a function of tuned LO frequency. These look-up tables were used in the design of standard science observations and were stored in the Common Uplink System (CUS). An identical copy of these lookup tables is contained in the calibration files associated with HIFI data downloaded from HSA. This is to enable checks whether the diplexer settings have differed from the intended ones.

As discussed in Section 2, the dual-sideband nature of HIFI implies that the OPD should be as close as possible to the nominal value of $\sim 25 \mathrm{~mm} / \sim 41.7 \mathrm{~mm}$ (bands $3 / 4$ and 6/7, respectively), while optimally coupling the LO. This results in a sawtooth curve (see Fig. 4). For LO frequencies not contained in the look-up table, the commanded values are interpolated. Interpolation is, of course, inappropriate at LO frequencies corresponding to the discontinuities of the sawtooth curve. Care was taken in the CUS design to prevent this from happening.

Diplexer repeatability The remaining diplexer-calibration observations were done to verify and quantify the repeatability of the HIFI diplexers. As discussed in Section 3, the first 6 repeat observations (QSFT 1-6) are identical repeats of the CoP observations, while QSFT 7-11 are shorter to save spacecraft time. These two sets of observations are analyzed separately.

Table 2 contains the fitted parameters per test: $d_{0}$ and $\beta$ (see Eq. 3) for each band and polarization. For each set of observations, the mean and standard deviation are given. Some parameters display a standard deviation of zero; this is due to the limited bit resolution of the DAC of the actuator current source. The uncertainty of the OPD, $\delta \mathrm{OPD}$, follows from the uncertainties of the fit parameters and Eq. 3 (neglecting the quadratic term):

$$
\left(\frac{\delta \mathrm{OPD}}{2}\right)^{2}=\left(\delta d_{0}\right)^{2}+\left(I_{\max } L \frac{\pi}{180^{\circ}} \delta \beta\right)^{2}
$$

where $I_{\max }=2 \mathrm{~mA}$ and $L=27.5 \mathrm{~mm}$. $\delta$ OPD is given in Table 2 .

Fig. 4 Visualization of the look-up table for actuator current as a function of tuned LO frequency (for band 3a)

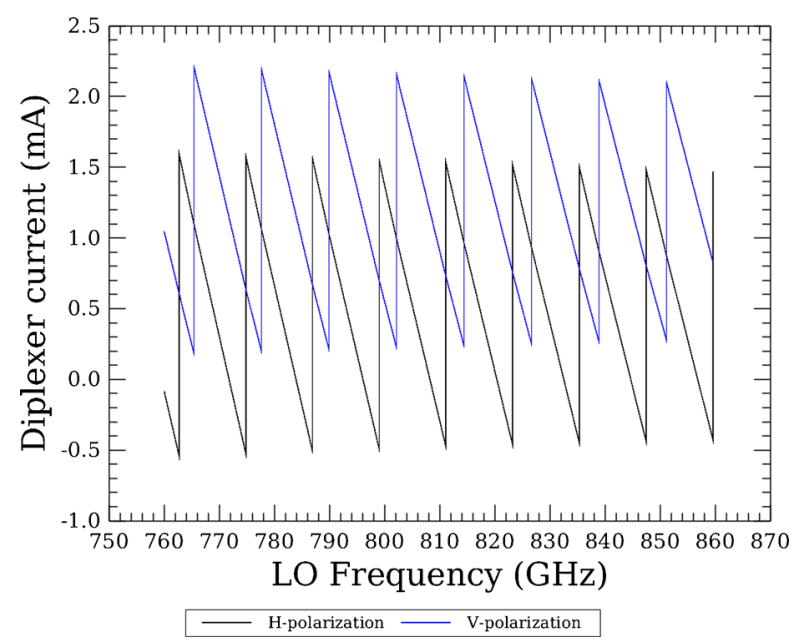


Influence on HIFI data quality A mistuned diplexer leads to signal loss through lessthan-optimum coupling, resulting in and amplified by non-optimum pumping of the mixers. Also, a mistuned diplexer would in general affect the upper and lower sideband differently, thus inducing a spectral dependence (slope) of the sideband-ratio (see [3]) over the IF.

The formal OPD uncertainties as given in Table 2 are generally at the sub- $\mu \mathrm{m}$ level, comparable to the step size of the actuators of $\sim 200 \mathrm{~nm}$, which provides a lower bound on the achievable OPD uncertainty. The OPD uncertainty is $0.31 \%$ of the observed wavelength or better. This demonstrates that the diplexers and their calibration were robust throughout the mission. The repeatability and precision of the tuning mechanism are so high that science data are in no way negatively affected. We caution that, using the data discussed, we cannot rule out systematic uncertainties in the diplexer calibration. While we are not aware of any, they would adversely impact HIFI data quality as discussed above.

We remark that a handful of science observations did suffer from an appreciably mistuned diplexer. In these unlucky cases, the commanded LO frequency fell within the "gap" of the look-up table around one of the discontinuities of the sawtooth curve (see Fig. 4). Despite safeguards in the CUS software, this is not completely avoidable due to the finite granularity of the table. The commanded actuator current was therefore interpolated to an inappropriate value, resulting in a mistuned diplexer (typically only in one of the two polarizations). All these cases were properly identified by the pipeline, and data-quality flags were raised as appropriate.

\section{Using diplexers to detect spectral impurities}

With the diplexer model established and validated as above, the line of reasoning can be reversed and the diplexers can be used as relative spectrometers to measure the LO frequency. This was used extensively, both pre-launch and in-flight, to identify ranges of LO frequency that were operated at non-optimal settings leading to spectral impurity, i.e., to a measurable fraction of the output power being emitted at a frequency different from that commanded. An independent approach to detecting impurities is through measurements of the IV curves of the multipliers within the LO [5].

Impurities were identified through dedicated surveys, where the LO was stepped through the entire frequency range of the diplexer bands at a step width of $2 \mathrm{GHz}$. At each LO frequency, a diplexer scan is performed as described above. The OPD is calculated from the actuator current using the validated diplexer model. The LO frequency is then calculated from the observed interference pattern and compared to the commanded LO frequency. Impurities are readily apparent as mismatch between the two (see, e.g., Fig. 9 in [1]). ${ }^{4}$

\footnotetext{
${ }^{4}$ Effectively, the diplexers act as Fourier-transform spectrometers, albeit with extremely low spectral resolution (the OPD excursion is only 2-7 wavelengths). Also, as detailed above, the diplexer calibration relies on that of the LO frequency, so this method cannot provide any absolute frequency measurements.
} 
Dedicated purification campaigns were run at identified impure LO frequencies. They consisted in running the diplexer scan for a range of LO settings (multiplier currents, bias voltages, ... ). This allowed the ICC to identify more appropriate LO settings, with good spectral purity, that were used for subsequent observations.

This way, the important CII line at $1901 \mathrm{GHz}$ (requiring an LO frequency of 1897$1898 \mathrm{GHz}$ in the upper sideband) was purified just months before launch (see [1]). Parts of band 7 were purified in flight. The region in band 3 with LO frequencies longward of $951 \mathrm{GHz}$ was purified in a dedicated campaign ending in March 2013, just before the end of Herschel's cryogenic lifetime.

Open Access This article is distributed under the terms of the Creative Commons Attribution License which permits any use, distribution, and reproduction in any medium, provided the original author(s) and the source are credited.

\section{References}

1. Dieleman, P., Teyssier, D., Klein, Th., et al.: Performance of HIFI in flight conditions, 20th International Symposium on Space Terahertz Technology 2009 (ISSTT 2009), pp. 63-68. Curran Associates Inc. (2010)

2. de Graauw, T., Helmich, F.P., Phillips, T.G., et al.: The Herschel-Heterodyne Instrument for the FarInfrared (HIFI), A\&A, 518, L4 (2010)

3. Higgins, R., Teyssier, D., Braine, J.: The effect of side band ratio on line intensity determination for the Herschel/HIFI spectrometer. Exp. Astron. submitted (2014)

4. Martin, D.H., Puplett, E.: Polarised interferometric spectrometry for the millimetre and submillimetre spectrum. Infrared Phys. 10, 105-109 (1969)

5. Pearson, J., Teyssier, D., Maiwald, F., Ward, J., Lin, R., Mehdi, I., Kooi, J., Klein, T., Leinz, C., Jellema, W., Risacher, C.: Verification of spectral purity in the HIFI local oscillator, 22nd International Symposium on Space Terahertz Technology, Tucson (2011)

6. Pilbratt, G.L., Riedinger, J.R., Passvogel, T., et al.: Herschel space observatory, an ESA facility for far-infrared and submillimetre astronomy, A\&A, 518, L1 (2010)

7. Roelfsema, P.R., Helmich, F.P., Teyssier, D., et al.: In-orbit performance of Herschel-HIFI, A\&A, 537, A17 (2012) 